Cita: Tristán, J.; Vergara-Torres, A.; Vanegas-Farfano, M.; Espino-Verdugo, F.; MedinaCorrales, M.; Tomás, I. (2019). Presentación de las tareas proporcionado por el profesor, necesidades psicológicas y vitalidad subjetiva en alumnos de educación física. Cuadernos de Psicología del Deporte, 19(3), 190-204

\title{
Presentación de las tareas proporcionado por el profesor, necesidades psicológicas y vitalidad subjetiva en alumnos de educación física
}

\section{Task presentation provided by the teacher, psychological needs and subjective vitality in physical education students}

\section{Apresentação das tarefas fornecidas pelo profesor, das necesidades psicológicas e da vitalidade subjetiva dos alunos de educação física}

\author{
Tristán, J. ${ }^{1}$, Vergara-Torres, A. ${ }^{1}$, Vanegas-Farfano, M. ${ }^{1}$ Espino-Verdugo, F. ${ }^{1}$, Medina-Corrales, M. , \\ Tomás, I. ${ }^{2}$ \\ ${ }^{1}$ Universidad Autónoma de Nuevo León; ${ }^{2}$ Universitat de València
}

\section{RESUMEN}

Tomando como base la teoría de las necesidades psicológicas básicas (Deci y Ryan, 2002), en este estudio se examinó si la percepción que tiene el alumno de la presentación de las tareas por parte del profesor predice la satisfacción de las necesidades psicológicas básicas, y éstas a su vez predicen el bienestar (vitalidad subjetiva) de los alumnos en la sesión de educación física. La muestra estuvo compuesta por 515 niños ( 272 hombres $[M=11.28$ años; $D T=.49]$ y 243 mujeres $[M=11.36$ años; $D T=.48])$ estudiantes de sexto grado de primaria $(M=11.32 ; D T=.48)$, que cumplimentaron los instrumentos que evaluaban las variables del estudio. Los resultados del análisis de ecuaciones estructurales indicaron que la presentación de las tareas actuó como predictor positivo de la satisfacción de las necesidades psicológicas básicas, y que éstas a su vez predijeron positivamente la vitalidad subjetiva. Los análisis de mediación indicaron que la satisfacción de las necesidades psicológicas básicas medió parcialmente la relación entre la percepción que tiene el alumno de la presentación de las tareas por el profesor y el bienestar psicológico de los alumnos de educación física.

Palabras clave: Instrucción pre-practica, Teoría de la autodeterminación, Vitalidad subjetiva, Profesor.

\section{ABSTRACT}

Within the framework of basic psychological needs theory (Deci \& Ryan, 2002), in this study we examined the students' perception of the coach's task presentation as a predictor of the satisfaction of the basic psychological needs, which in turn predicted psychological wellbeing (subjective vitality) of students in the physical education context. Participants were 515 children $(272$ men $[M=11.28$ years; $S D=.49]$ and 243 women $[M=11.32$ years; $S D$ $=.48])$ students in the sixth grade of primary school $(M=11.32$ years, $S D=.48)$, who completed the questionnaires measuring the study variables. Results of structural equation analyses showed that coach's task presentation positively predicted satisfaction of the basic psychological needs, which in turn positively predicted subjective vitality. Satisfaction of the basic psychological needs partially mediated the relationship between coach's task presentation and well-being of students in the physical education context.

Keywords: Pre-practical instruction; Self-determination theory; Subjective vitality, Teacher. 


\section{Presentación de las tareas, necesidades psicológicas y vitalidad subjetiva en la educación física}

\section{RESUMO}

Tendo como base a teoria das necessidades psicológicas básicas (Deci e Ryan, 2002), este estudo busca analisar se a percepção predefinida pelos alunos sobre a apresentação das atividades propostas pelo professor predizem à satisfação das necessidades psicológicas básicas, e estas por sua vez, definem o bem-estar (vitalidade subjetiva) dos alunos na aula de educação física. A amostra está conformada por 515 crianças ( 272 meninos $[M=11.28$ anos; $D T$ $=.49]$ e 243 meninas $[M=11.32$ anos; $D T=.48])$ estudantes de sexto ano da primária $(M=11.32$ anos; $D T=.48)$, que preencheram os instrumentos de avaliação das variáveis do estudo. Os resultados da análise de equações estruturais mostraram que a apresentação das atividades atuou como influenciador positivo da satisfação das necessidades psicológicas básicas e que estas, por sua vez, manifestaram-se positivamente frente a vitalidade subjetiva. As análises de mediação indicaram que a satisfação das necessidades psicológicas básicas mediu parcialmente a relação entre a percepção do aluno sobre a apresentação das tarefas pelo professor e o bem-estar psicológico dos alunos de educação física.

Palavras chave: Ensino pré-prático, Teoria da autodeterminação, Vitalidade subjetiva, Professor.

\section{INTRODUCCIÓN}

La clase de educación física constituye un espacio donde se generan ambientes de aprendizaje en el que niños y adolescentes pueden desarrollar sus capacidades cognitivas, psicomotrices y socioafectivas (McLennan y Thompsom, 2015). El responsable de generar dichos ambientes es el profesor de educación física, quien a través de su intervención en la clase se convierte en un factor clave en el logro de los objetivos de aprendizaje y el bienestar de los alumnos (Behzadnia, Adachi, Deci y Mohammadzadeh, 2018; Chen, Mason, Staniszewski, Upton y Valley, 2012; Rink y Hall, 2008). Estudios previos han identificado a la presentación de las tareas como uno de los ámbitos de intervención del profesor en las clases de educación física (Chen et al., 2012; Chen, Zhu, Mason, Hammond-Bennett y ColomboDougovito, 2016), y como un aspecto importante que impacta en la salud psicológica de los alumnos y no solamente como un aspecto pedagógico que busca el logro del aprendizaje (Garza-Adame, Tristán, Tomás, Hernández-Mendo y López-Walle, 2017). Por eso, estudiar la presentación de las tareas por parte del profesor desde una perspectiva de la Teoría de la Autodeterminación (Self-Determination Theory, SDT; Ryan y Deci 2017) es relevante y necesario.

\section{Presentación de las tareas por parte del profesor de educación física}

En las últimas dos décadas, los estudios sobre el comportamiento de los profesores (e.g. Cuevas, García-López y Contreras, 2015; Trigueros-Ramos, Navarro-Gómez, Aguilar-Parra y León-Estrada, 2019) se han realizado cada vez más desde la perspectiva de la SDT (Ryan y Deci, 2017). Investigaciones realizadas sobre el comportamiento del profesor, en específico sobre la presentación de las tareas (Chen et al., 2012, 2016; Hall, Heirdon y Welch, 2011), han permitido concluir que la presentación de las tareas de calidad son un factor que contribuye a la enseñanza efectiva y, a su vez, al aprendizaje de los estudiantes (Hall et al., 2011), es decir, es una de las variables predictoras de la eficacia pedagógica, que se puede definir como la búsqueda de resultados de aprendizaje, el diseño de experiencias de aprendizaje con la intensión de lograr dichos resultados y la evaluación de hasta qué punto se han conseguido (Rink y Hall, 2008).

La presentación de las tareas (Rink, 2019) o estructura antes de la actividad (Tristán et al., 2016) es definida como la comunicación del profesor hacia los alumnos de qué hacer y cómo hacerlo antes de iniciar la práctica de las tareas o actividades (Rink, 1994) y engloba las intervenciones verbales y no verbales del profesor, como la explicación y demostración de un movimiento o gesto técnico (Chen et al., 2012; Kelly y Melograno, 2004; Piéron, 1996; Rink, 2013; Tristán et al., 2016). Una presentación de tareas de calidad necesita tener un enfoque cognitivo para mejorar las habilidades de los estudiantes (Magill, 1993; Rink, 2019). El enfoque cognitivo se presenta con un énfasis en los elementos cualitativos críticos del movimiento deseado, que son comunicados a través de claves de aprendizaje cualitativas precisas, en forma concisa, y con un número apropiado para el alumno (Chen et al., 2012; Landin, 1994; Masser, 1993; Rink, 2019). Al comunicar expectativas claras y al enmarcar la actividad de aprendizaje de los estudiantes con instrucciones y orientación explícitas, estos actos de 


\section{Tristán, J., Vergara-Torres, A., Vanegas-Farfano, M., Espino-Verdugo, F., Medina-Corrales, M., Tomás, I.}

instrucción apoyan el compromiso de los estudiantes al mantener a estos en la tarea, controlando su comportamiento y evitando el caos durante las transiciones (Skinner y Belmont, 1993; Tucker et al., 2002).

Estudios anteriores (Chen, Hendricks y Archibald, 2011; Chen, Mason, Hammond-Bennett y Zlamout, 2014; Gusthart y Kelly, 1993; Gusthart, Kelly y Rink, 1997; Hall et al., 2011; Sau-Ching, 2001) han demostrado que los profesores eficaces realizan una presentación de las tareas que se caracteriza por todos o la mayoría de los siguientes elementos: 1) utilizan demostraciones completas; 2) tienen un enfoque cognitivo usando claves que son apropiadas en número, precisas y de valor cualitativo; 3 ) son claras para los alumnos; y 4) el feedback es congruente con el enfoque cognitivo.

Otros aspectos a considerar en las presentaciones de tareas efectivas, consisten en la comunicación del enfoque o intención de la actividad; del significado e importancia de lo que se va a aprender así como de la estructura organizativa de la clase; proporcionar ejemplos negativos de lo que se pretende, resumir la información y repetición de las expectativas, dar a los alumnos opciones entre las tareas, y en general brindar a los estudiantes una guía para la toma de decisiones en la clase (Chen et al., 2012; Kwak, 2005; Graham, 1988; Gusthart et al., 1997; Hall et al., 2011; Rink, 1994, 2019). La presencia de estas características en una presentación de las tareas determina su calidad.

Sin embargo, si se considera la presentación de las tareas o estructura antes de la tarea desde una perspectiva psicológica y tomando en cuenta los resultados de investigaciones anteriores (Curran, Hill y Niemiec, 2013; Deci y Ryan, 2002; Garza-Adame et al., 2017; Reeve y Jang, 2006; Tristán et al., 2016) se podría señalar que al momento de presentar la tarea los profesores podrían considerar: explicar la relevancia de las actividades de aprendizaje; proporcionar expectativas claras; mostrar un interés sincero en las preferencias de los alumnos y escucharlos de forma activa; crear oportunidades para que los alumnos tomen la iniciativa; conceder al alumno toma de decisiones; proveer ayuda a los alumnos durante las actividades; interactuar con los alumnos de una manera cálida, amistosa y afectuosa.

\section{Satisfacción de las necesidades psicológicas básicas y el bienestar psicológico de los alumnos}

Una de las mini-teorías en el marco de la SDT es la teoría de las necesidades psicológicas básicas (BPNT, Basic Psychological Needs Theory; Deci y Ryan, 2002). De acuerdo a esta teoría se asume que el ser humano posee las necesidades innatas y universales de percepción de competencia, autonomía y relación. La necesidad de autonomía se refiere a la necesidad de elegir cuándo y cómo regular la conducta y refleja el deseo de elegir por uno mismo. El comportamiento autónomo no sólo es intencional, sino también volitivo, y los individuos están totalmente de acuerdo en comprometerse en sus acciones (Deci y Ryan, 2002; Ryan y Deci, 2000). Para favorecer la satisfacción de esta necesidad es necesario que los profesores: expliquen la relevancia de las actividades de aprendizaje (Curran, Hill y Niemiec, 2013; Deci y Ryan, 2002; Garza-Adame et al., 2017; Reeve y Jang, 2006; Tristán et al., 2016); comuniquen el por qué van a realizar cada una de las actividades y la relación que tienen con la sesión anterior (Garza-Adame et al., 2017); muestren un interés sincero en las preferencias de los alumnos y los escuchen de forma activa (Curran et al., 2013; Garza-Adame et al., 2017; Haerens et al., 2013; Tristán et al., 2016); y creen oportunidades para que los alumnos tomen la iniciativa, además de conceder autonomía en la toma de decisiones (Moreno-Murcia, Cervelló, Montero, Vera y García, 2012; Reeve y Jang, 2006).

La necesidad de competencia se refiere a la necesidad de ser eficiente en las interacciones con el entorno y refleja el deseo que tienen las personas por ejercitar sus capacidades y habilidades propias (Deci y Ryan, 2002; Ryan y Deci, 2000). Para favorecer que los estudiantes desarrollen un sentido de eficacia el profesor debe: proporcionar expectativas claras (Deci y Ryan, 2002; Garza-Adame et al., 2017; Haerens et al., 2013; Tristán et al., 2016); especificar el objetivo de la tarea, explicar y demostrar cómo lograr tener éxito en la actividad o facilitar cómo realizar y ser competentes en la misma (Garza-Adame et al., 2017; Smith et al., 2015; Tristán et al., 2016); y proveer ayuda a los alumnos durante las actividades (Jang, Reeve y Deci, 2010).

La necesidad de relación se refiere a la necesidad de establecer lazos emocionales cercanos con otras personas y experimentar un sentido de conexión con los demás. La necesidad de relación incluye un 


\section{Presentación de las tareas, necesidades psicológicas y vitalidad subjetiva en la educación física}

sentimiento de ser incluido y cuidado por otros dentro del dominio de la acción, y un sentido de pertenencia a un entorno social (Deci y Ryan, 2002; Ryan y Deci, 2000). Para favorecer la satisfacción de esta necesidad, es importante que el profesor: brinde orientación e instrucción a todos los alumnos (Deci y Ryan, 2002; Haerens et al., 2013; Smith et al., 2015; Tristán et al., 2016); tome en consideración a todos los alumnos, mencione a los alumnos por su nombre en la explicación y demostración de las tareas, vaya rotando a los participantes con los que se apoya para las demostraciones, y pregunte a todos si comprendieron las tareas (Garza-Adame et al., 2017); y presente las tareas e interactúe con los alumnos de una manera cálida, amistosa y afectuosa (Cox y Williams, 2008; Soenens, Duriez, Vansteenkiste y Goossens, 2007).

En línea con la SDT, el cumplimiento de estas necesidades se corresponde con la satisfacción de las necesidades psicológicas básicas y la mejora del bienestar psicológico (Deci y Ryan, 2000) como la vitalidad subjetiva (Ryan y Deci, 2002). Esta teoría también propone que el desarrollo del bienestar o del malestar está en función del medio social (por ejemplo, el comportamiento de los profesores de educación física) y de su potencial para la satisfacción de las necesidades psicológicas básicas de los estudiantes (por ejemplo, para la participación en la educación física). La vitalidad subjetiva se refiere a un sentimiento positivo de tener una experiencia consciente de vitalidad y energía percibida que emana del yo (Ryan y Frederick, 1997). En otras palabras, se trata de una experiencia psicológica específica de tener energía positiva disponible o dentro del control regulador de uno mismo, energía que posee entusiasmo y voluntad, y representa "un indicador significativo del bienestar personal" (Vlachopoulos et al., 2011).

Diferentes investigaciones en el contexto del deporte han encontrado una relación positiva entre la estructura o la presentación de las tareas de los entrenadores y la satisfacción de las necesidades psicológicas básicas (Curran et al., 2013; GarzaAdame et al., 2017; Smith et al., 2015; Tristán et al., 2016). En el estudio realizado por Curran et al. (2013) señalan que el suministro de información, expectativas, estrategias, apoyo, límites y otros aspectos de la estructura no son necesariamente inhibidores de la participación volitiva en el deporte. Este estudio también indica que la comunicación de la estructura en forma de apoyo a la autonomía satisface las necesidades psicológicas básicas. En la investigación realizada por Smith et al. (2015) hallaron que la estructura (presentación de las tareas por parte del entrenador) predecía positivamente la satisfacción de la necesidad de autonomía, competencia y relación. En el estudio realizado por Tristán et al. (2016) encontraron que la presentación de las tareas tiene una relación positiva y está fuertemente asociada con la percepción de un clima motivacional de apoyo a la autonomía por parte del entrenador, y está débilmente asociada con los entrenadores que son percibidos como controladores (clima motivacional de estilo controlador). También, encontraron que la presentación de las tareas tiene una relación positiva y fuertemente asociada con la satisfacción de las necesidades psicológicas básicas y débilmente asociada con la frustración de las mismas. Los investigadores sugieren que cuando los entrenadores dan instrucciones verbales claras, demuestran actividades, proporcionan una justificación para sus solicitudes y recomendaciones y consideran las perspectivas de los jugadores, se generan condiciones que son propicias para la satisfacción de las necesidades psicológicas básicas de los atletas. Por otra parte, los resultados del estudio realizado por Garza-Adame et al. (2017) demuestran que los entrenadores que presentan la tarea con calidad, logran satisfacer las necesidades de autonomía, competencia y relación de los atletas, que la satisfacción de las tres necesidades psicológicas básicas fueron predictoras positivas de la vitalidad subjetiva, y que la satisfacción de las necesidades psicológicas media entre la presentación de las tareas y el bienestar de los atletas (expresado a través de su percepción de vitalidad).

Las relaciones positivas postuladas por la SDT entre la satisfacción de las necesidades psicológicas básicas y el bienestar han sido estudiadas por diferentes investigadores en el contexto deportivo, utilizando como indicador la variable que se utiliza en la presente investigación, esto es, la vitalidad subjetiva (e.g., Adie, Duda y Ntoumanis, 2008; Balaguer y Castillo, 2007; Reinboth y Duda, 2006; Reinboth, Duda y Ntoumanis, 2004). En los estudios realizados por Adie et al. (2008) y Garza-Adame et al. (2017), estos autores encontraron que la satisfacción de cada una de las tres necesidades psicológicas básicas actuaba como predictor de la vitalidad subjetiva. Sin embargo, en el estudio realizado por Reinboth et al. (2004) la satisfacción de la necesidad de relación no actuó como 


\section{Tristán, J., Vergara-Torres, A., Vanegas-Farfano, M., Espino-Verdugo, F., Medina-Corrales, M., Tomás, I.}

predictor del bienestar; de manera similar, en el estudio de Reinboth y Duda (2006) la satisfacción de la necesidad de competencia tampoco predijo el bienestar. Por otra parte, Balaguer y Castillo (2007), encontraron que la satisfacción de las necesidades psicológicas básicas en su conjunto, actuaron como predictoras de la vitalidad subjetiva. Garza-Adame et al. (2017) también encontraron que la satisfacción de las necesidades psicológicas media entre la presentación de las tareas y el bienestar de los atletas. Es decir, que el mecanismo que media entre la presentación de las tareas con calidad y el bienestar de los deportistas, es la satisfacción de las necesidades psicológicas básicas, ya que se consigue que los atletas se sientan autónomos, competentes y relacionados.

Hasta aquí es evidente que la secuencia de relaciones hipotetizadas en este estudio ha sido puesta a prueba en el contexto deportivo universitario. ¿Pero qué sabemos respecto al contexto de la educación física? A pesar de que el ámbito deportivo y la educación física comparten características comunes, son distintos y tienen atributos únicos (Tristán et al., 2016). Mientras que en el contexto de la enseñanza deportiva los objetivos pueden centrarse al desarrollo de habilidades propias de la disciplina deportiva practicada (Abad, Benito, Giménez y Robles, 2013), la educación física se enfoca en objetivos motrices, cognitivos y socioafectivos para llevar una vida saludable y de bienestar (Bailey, 2006). En ese sentido, Vlachopoulos et al. (2011) consideran que es necesario realizar estudios en la educación física utilizando la satisfacción de las necesidades psicológicas básicas ya que, aunque existen algunos estudios, estos son escasos. Concretamente, las relaciones entre la satisfacción de las necesidades psicológicas básicas y la vitalidad subjetiva han sido estudiadas por diferentes investigadores en el contexto de la educación física (e.g., Taylor y Lonsdale, 2010; Vlachopoulos et al., 2011). En el estudio realizado por Taylor y Lonsdale (2010) hallaron que la relación entre el clima motivacional de apoyo a la autonomía y la vitalidad subjetiva estaba mediada por las percepciones de los estudiantes de la satisfacción de sus necesidades psicológicas básicas. Por su parte, Vlachopoulos et al. (2011) encontraron que cada una de las tres necesidades psicológicas básicas mediaba entre el clima motivacional de apoyo a la autonomía del profesor de educación física y los niveles de vitalidad subjetiva que experimentaron los alumnos de educación física.
Hasta la fecha no se han encontrado investigaciones realizadas con alumnos de educación física en México donde sea analizada la secuencia de la presentación de las tareas por parte del profesor, la satisfacción de las necesidades psicológicas básicas y el bienestar, por lo que consideramos que este estudio contribuye a probar empíricamente la BPNT con la presentación de las tareas en el contexto de la educación física.

El objetivo principal de esta investigación fue estudiar, desde los planteamientos de la miniteoría de la BPNT (Deci y Ryan, 2002), las interrelaciones entre la presentación de las tareas (PT) por parte del profesor como factor social, las necesidades psicológicas básicas (NPB: autonomía, competencia y relación) como factores mediadores personales, y la vitalidad subjetiva (VS) en el contexto de la educación física. Todo esto, a través de la comprobación de un modelo teórico con la secuencia PT $\rightarrow \mathrm{NPB} \rightarrow \mathrm{VS}$ (fig. 1), en el que se plantean las siguientes hipótesis: 1) la presentación de las tareas por parte del profesor será un predictor positivo de la satisfacción de las necesidades psicológicas básicas; 2) la satisfacción de NPB será un predictor positivo de la vitalidad subjetiva, y 3) la satisfacción de las NPB mediará la relación entre la presentación de las tareas por parte del profesor y la vitalidad subjetiva descrita por el alumno. Aunque esta relación ha sido estudiada en el contexto del deporte (e.g., Garza-Adame et al., 2017), la investigación es escasa en el contexto de la educación física, y la necesidad de su estudio ha sido puesta de manifiesto (e.g., Vlachopoulos, Katartzi y Kontou, 2011).

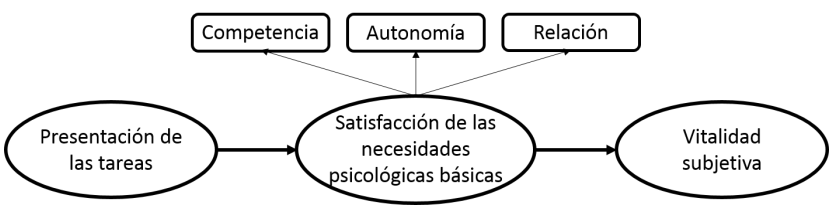

Figura 1. Representación del modelo estructural hipotetizado.

\section{MATERIAL Y MÉTODOS}

\section{Participantes}

La selección de los participantes se realizó mediante un muestreo por conveniencia. La muestra estuvo compuesta por 515 niños (272 hombres $[M=11.28$ años; $D T=.49]$ y 243 mujeres $[M=11.36$ años; $D T$ $=.48]$ ) estudiantes de sexto grado de primaria de diferentes escuelas urbanas públicas del estado de 


\section{Presentación de las tareas, necesidades psicológicas y vitalidad subjetiva en la educación física}

Nuevo León, México. Se seleccionaron alumnos de sexto grado para mejor compresión de las instrucciones y los ítems de los instrumentos. Los participantes del estudio tenían edades comprendidas entre los 10 y 13 años $(M=11.32 ; D T=.48)$, y tenían entre una y dos clases de educación física por semana $(M=1.61 ; D T=.48)$, con una duración de entre $40 \mathrm{y}$ 60 minutos por sesión $(M=45.57 ; D T=5.66)$.

\section{Instrumentos}

En la tabla 1, se presentan los instrumentos utilizados para medir las variables de estudio, sus características y validaciones.

Las fiabilidades de los instrumentos han sido probadas en estudios previos. En el caso del EPTE, el estudio de Garza-Adame et al. (2017) ha comprobado su fiabilidad en el contexto deportivo, obteniendo un coeficiente alpha de Cronbach de .93. En este estudio, la fiabilidad de la escala evaluada mediante el coeficiente alpha de Cronbach fue de .83, resultando por tanto satisfactoria. En la tabla 2 se presenta la versión adaptada al contexto de la educación física de esta escala así como sus estadísticos descriptivos. La fiabilidad de la Escala Mexicana de Satisfacción de las Necesidades Psicológicas Básicas fue comprobada por sus autores (Zamarripa et al., 2017) y confirmada en este estudio, obteniendo un valor alpha de Cronbach de .85. En el caso de la vitalidad subjetiva (indicador del bienestar psicológico) la fiabilidad de la Escala de Vitalidad Subjetiva ha sido confirmada en el contexto deportivo (Garza-Adame et al., 2017), en los datos de este estudio, el alpha de Cronbach de la escala fue de .78, resultando por tanto satisfactoria.

En las adaptaciones de los instrumentos al contexto de la educación física de nivel primaria, participaron profesores de educación física e investigadores especialistas en el área.

\section{Procedimiento}

Para la aplicación de los instrumentos, se solicitó autorización a los directores de las escuelas participantes y se les proporcionó un ejemplar del instrumento. Todos los participantes y sus padres recibieron información verbal sobre la investigación y dieron su consentimiento informado antes de la recopilación de los datos. Los cuestionarios fueron contestados de forma anónima y voluntaria y fueron administrados por al menos un investigador quien explicó a los participantes que no existían respuestas correctas e incorrectas así como que las respuestas fueran realizadas con completa honestidad. La administración de los instrumentos se realizó en un horario regular de clases y tuvo una duración aproximada de 25 minutos, en la cual, el profesor de educación física no estuvo presente en ningún momento.

Tabla 1

Instrumentos de medición de variables de estudio.

\begin{tabular}{|c|c|c|c|c|}
\hline Variable & Instrumento & Validado por & Ítems & Ejemplo de ítem \\
\hline $\begin{array}{l}\text { Presentación de } \\
\text { las tareas }\end{array}$ & $\begin{array}{l}\text { Versión adaptada de la } \\
\text { Escala de Presentación de } \\
\text { las Tareas del Entrenador } \\
\text { (EPTE) }\end{array}$ & $\begin{array}{l}\text { Tristán et al. } \\
\text { (2016) }\end{array}$ & $\begin{array}{l}11 \text { ítems con } \\
\text { respuesta tipo } \\
\text { Likert de } 1 \text { a } 5\end{array}$ & $\begin{array}{l}\text { "mi profesor(a) me dice } \\
\text { en qué elementos } \\
\text { técnicos enfocarme para } \\
\text { realizar correctamente la } \\
\text { actividad, tarea o } \\
\text { ejercicio" }\end{array}$ \\
\hline $\begin{array}{l}\text { Satisfacción de } \\
\text { necesidades } \\
\text { psicológicas } \\
\text { básicas }\end{array}$ & $\begin{array}{l}\text { Escala Mexicana de } \\
\text { Satisfacción de las } \\
\text { Necesidades Psicológicas } \\
\text { Básicas en Educación } \\
\text { Física }\end{array}$ & $\begin{array}{l}\text { Zamarripa, } \\
\text { Castillo, Tomás, } \\
\text { Delgado y } \\
\text { Álvarez (2017) }\end{array}$ & $\begin{array}{l}16 \text { ítems con } \\
\text { respuesta tipo } \\
\text { Likert de } 1 \text { a } 7\end{array}$ & $\begin{array}{l}\text { "mi opinión cuenta en } \\
\text { cuanto a qué actividades } \\
\text { quiero practicar" }\end{array}$ \\
\hline $\begin{array}{l}\text { Vitalidad } \\
\text { subjetiva }\end{array}$ & $\begin{array}{l}\text { Versión adaptada de la } \\
\text { Escala de Vitalidad } \\
\text { Subjetiva }\end{array}$ & $\begin{array}{l}\text { López-Walle, } \\
\text { Balaguer, } \\
\text { Castillo y } \\
\text { Tristán (2012) }\end{array}$ & $\begin{array}{l}6 \text { ítems con } \\
\text { respuesta tipo } \\
\text { Likert de } 1 \text { a } 7\end{array}$ & $\begin{array}{l}\text { "me siento con ánimo y } \\
\text { entusiasmo (vivo) y lleno } \\
\text { de vida (vital)" }\end{array}$ \\
\hline
\end{tabular}




\section{Tristán, J., Vergara-Torres, A., Vanegas-Farfano, M., Espino-Verdugo, F., Medina-Corrales, M., Tomás, I.}

Tabla 2

Estadísticos descriptivos de la Versión adaptada de la EPTE

\begin{tabular}{|c|c|c|c|c|}
\hline Ítem & $M$ & $D T$ & Asimetría & Curtosis \\
\hline $\begin{array}{l}\text { 1. Mi profesor(a) me dice en qué poner más atención para realizar } \\
\text { correctamente la actividad, tarea o ejercicio. }\end{array}$ & 4.11 & 0.96 & -1.22 & 1.44 \\
\hline $\begin{array}{l}\text { 2. Mi profesor(a) me dice en qué enfocarme para hacer bien los movimientos } \\
\text { de la actividad, tarea o ejercicio. }\end{array}$ & 4.13 & 0.89 & -1.01 & 0.97 \\
\hline $\begin{array}{l}\text { 3. Mi profesor(a) me demuestra cómo no se deben de hacer los movimientos, } \\
\text { utilizando a un compañero o algún otro medio visual como ejemplo. }\end{array}$ & 4.06 & 1.07 & -1.10 & 0.65 \\
\hline $\begin{array}{l}\text { 4. Mi profesor(a) menciona el objetivo de cada una de las tareas, actividades } \\
\text { o ejercicios que se van a realizar. }\end{array}$ & 4.10 & 1.04 & -1.15 & 0.89 \\
\hline $\begin{array}{l}\text { 5. Mi profesor(a) me pide demasiados objetivos en los que debo poner } \\
\text { atención para hacer la actividad, tarea o ejercicio. }\end{array}$ & 3.51 & 1.13 & -0.49 & -0.29 \\
\hline $\begin{array}{l}\text { 6. Mi profesor(a) me explica verbalmente el movimiento, tarea ejercicio o } \\
\text { actividad que se va a realizar. }\end{array}$ & 4.23 & 0.89 & -1.19 & 1.42 \\
\hline $\begin{array}{l}\text { 7. Mi profesor(a) me dice de manera precisa en qué poner más atención en el } \\
\text { ejercicio que se va a realizar, mostrándonos que de esa forma el ejercicio } \\
\text { se puede realizar con menos esfuerzo. }\end{array}$ & 3.93 & 1.08 & -0.89 & 0.23 \\
\hline $\begin{array}{l}\text { 8. Mi profesor(a) me informa sobre los elementos (colocación de manos, } \\
\text { pies, etc.) de calidad en los que me tengo que enfocar al realizar un } \\
\text { movimiento. }\end{array}$ & 4.17 & 0.92 & -1.00 & 0.64 \\
\hline $\begin{array}{l}\text { 9. Mi profesor(a) me demuestra la forma correcta de hacer un movimiento } \\
\text { utilizando a un compañero o algún otro medio visual como ejemplo. }\end{array}$ & 4.20 & 0.95 & -1.28 & 1.57 \\
\hline 10. Mi profesor(a) me explica claramente qué y cómo hacer las cosas. & 4.35 & 0.83 & -1.42 & 2.25 \\
\hline $\begin{array}{l}\text { 11. Mi profesor(a) me demuestra visualmente la forma de hacer un } \\
\text { movimiento utilizando a un compañero o algún otro medio visual como } \\
\text { ejemplo. }\end{array}$ & 4.22 & 0.94 & -1.15 & 0.94 \\
\hline
\end{tabular}

\section{Análisis de datos}

Se realizaron análisis descriptivos de cada una de las variables del estudio (rango, media, desviación típica, curtosis y asimetría), y se realizó la prueba de Kolmogorov-Smirnov con el propósito de evaluar la normalidad de los datos. Se utilizó la correlación de Pearson para analizar las interrelaciones entre las variables del modelo y establecer el grado de asociación entre ellas. Los análisis descriptivos y correlacionales se realizaron mediante el software estadístico SPSS 24.0. También se llevaron a cabo análisis factoriales confirmatorios (AFCs) con el objetivo evaluar la estructura factorial de las escalas utilizadas en el estudio. Finalmente, para poner a prueba el modelo de mediación hipotetizado (figura 1), se realizó un análisis de ecuaciones estructurales (SEM). Se utilizó el software AMOS 24.0 en la realización de los AFCs y la evaluación del modelo hipotetizado mediante SEM. En ambos análisis, se tomaron en cuenta los siguientes índices de bondad de ajuste: ji-cuadrado partido por grados de libertad, el índice de ajuste comparativo (CFI, Comparative Fit Index) y la raíz del promedio del error de aproximación (RMSEA, Root mean Square Error of Aproximation) (Bentler, 1990; Bentler y Bonett, 1980; Hu y Bentler, 1995; Steigner y Lind, 1980; Wheaton, Muthén, Alwin y Summers, 1977). Se utilizaron los siguientes criterios para valorar el ajuste de los modelos: un cociente $\chi^{2 / g l}$ menor a 3 es indicador de ajuste satisfactorio (Carmines y McIver, 1981); para CFI valores superiores a .90 indican un ajuste aceptable (Hu y Bentler, 1995); en el caso de la RMSEA, valores inferiores a .08 indican un error razonable, mientras que valores superiores .10 no son admisibles (Browne y Cudeck, 1993). Para finalizar, en la evaluación del efecto de mediación o efecto indirecto puesto a prueba en el modelo, se utilizó el método bootstrap estimando un intervalo de confianza corregido de sesgo (bias-corrected boostrap confidence interval method; MacKinnon, Lockwood, y William, 2004; William y MacKinnon, 2008). Este 


\section{Presentación de las tareas, necesidades psicológicas y vitalidad subjetiva en la educación física}

método implica el cálculo del producto de los coeficientes de regresión que estiman el efecto indirecto $\left(b_{1} b_{2}\right)$, donde $b_{1}$ es el coeficiente que estima la relación entre la presentación de las tareas por parte del profesor y la satisfacción de las NPB, y $b_{2}$ es el coeficiente que estima la relación entre la satisfacción de las NPB y la vitalidad subjetiva. Posteriormente, se obtiene el intervalo de confianza de bootstrap para el efecto indirecto $\left(b_{1} b_{2}\right)$. Si el intervalo de confianza no incluye el valor cero, se obtiene evidencia empírica del efecto indirecto (es decir, del efecto de mediación). En cuanto a los criterios para interpretar los índices de ajuste incremental, Cheung y Rensvold (2002) sugieren que una diferencia de $.01 \mathrm{o}$ menos, entre los valores de CFI $(\triangle \mathrm{CFI}<.01)$ indica diferencias prácticamente irrelevantes entre los modelos. De manera similar, Chen (2007) sugirió que los aumentos de RMSEA $<.015$ entre modelos alternativos indican diferencias irrelevantes $\mathrm{y}$, por lo tanto, se debe seleccionar el modelo más parsimonioso.

\section{RESULTADOS}

\section{Estadísticos descriptivos}

En la tabla 3 se presentan los estadísticos descriptivos de las variables del estudio, los cuales indican que los valores obtenidos se encuentran dentro de los parámetros de normalidad. De acuerdo con la prueba de Kolmogorov-Smirnov los resultados en todas las variables de estudio no fueron significativos $(p>.05)$, lo que confirma una distribución normal de los datos. Los coeficientes de fiabilidad de todas las escalas fueron satisfactorios (entre .78 y .85), estando por encima del criterio de .70 establecido por Peterson (1994). En el análisis de interrelación entre las variables de estudio se aprecia una interrelación positiva y significativa entre todas ellas. $\mathrm{La}$ presentación de las tareas por parte del entrenador presentó una correlación positiva y significativa con las necesidades psicológicas básicas en su conjunto (competencia, autonomía y relaciones), así como con la vitalidad subjetiva. Además, las necesidades psicológicas básicas se correlacionaron de manera positiva y significativa con la vitalidad subjetiva.

\section{Análisis factoriales confirmatorios}

Los análisis factoriales confirmatorios de las escalas se realizaron empleando el método de máxima verosimilitud, ya que como se ha comentado en el apartado anterior, los datos presentaban distribución normal.

En la tabla 4 se muestran los índices de bondad de ajuste de los modelos de medida unifactoriales para cada una de las escalas y, por lo tanto, se ofrecen evidencias de validación de cada una de las variables en la muestra de este estudio. Todas las escalas presentaron índices de ajuste satisfactorios.

\section{Modelo hipotetizado}

El modelo de mediación hipotetizado (ver figura 1) presentó índices de ajuste adecuados $\left(\chi^{2}(475)=\right.$ 915.787, $\chi^{2} / g l=1.928, \mathrm{CFI}=.936$, RMSEA $\left.=.042\right)$. En la figura 2 se presenta la solución estandarizada del modelo estructural. De acuerdo con los resultados obtenidos la presentación de las tareas por parte del profesor de educación física resultó ser un predictor positivo y significativo de la satisfacción de las necesidades psicológicas básicas en su conjunto $\left(\beta_{I}=.64, p<.001\right)$ y estás, a su vez, fueron predictoras de la vitalidad subjetiva $\left(\beta_{2}=.70, p<.001\right)$, que es indicador del bienestar psicológico de los alumnos de educación física. Además, el intervalo de confianza (IC) estimado por bootstrap para el efecto indirecto no incluyó el valor cero $\left(b_{1} b_{2}=.39\right.$; IC $95 \%=$ $[.168, .649])$, por lo que podemos concluir que la satisfacción de las NPB medió la relación entre la presentación de las tareas por parte del profesor y la vitalidad subjetiva.

Tabla 3

Estadísticos descriptivos, consistencia interna, normalidad y correlación entre las variables de estudio

\begin{tabular}{lccccccccc}
\hline & Rango & $M$ & $D T$ & $\alpha$ & Asimetría & Curtosis & $K-S$ & 1 & 2 \\
\hline 1 Presentación de las tareas & $1-5$ & 4.09 & 0.59 & .83 & -.77 & .53 & .09 & - & \\
2 Satisfacción de necesidades & $1-7$ & 5.06 & 0.94 & .85 & -.46 & .02 & .05 & $.44^{* *}$ & - \\
psicológicas básicas & $1-7$ & 5.29 & 1.07 & .78 & -.81 & .43 & .10 & $.43^{* *}$ & $.48^{* *}$ \\
\hline
\end{tabular}

Nota: $M=$ Media, $D T=$ Desviación estándar, $\alpha=$ Alpha de Cronbach, $K-S=$ Kolmogorov-Smirnov; $* * p<.01$ 


\section{Tristán, J., Vergara-Torres, A., Vanegas-Farfano, M., Espino-Verdugo, F., Medina-Corrales, M., Tomás, I.}

Tabla 4

Índices de bondad de ajuste de los instrumentos

\begin{tabular}{lccccc}
\hline Factores latentes & $\chi 2$ & $g l$ & $\chi 2 / g l$ & CFI & RMSEA \\
\hline Presentación de las tareas & 86.48 & 35 & 2.47 & .96 & .05 \\
Satisfacción de las necesidades psicológicas básicas & 258.61 & 92 & 2.81 & .95 & .05 \\
Vitalidad subjetiva & 14.67 & 7 & 2.09 & .99 & .04 \\
\hline
\end{tabular}

Finalmente, para analizar el tipo de mediación (total o parcial), se comparó el ajuste del modelo hipotetizado de mediación total (M1) con un modelo alternativo de mediación parcial (M2) en el que se añadió un path que representaba el efecto directo de la presentación de las tareas por parte del profesor sobre la vitalidad subjetiva (ver figura 3). Para comparar los dos modelos alternativos, se calculó la diferencia de jicuadrado entre los dos modelos anidados, y adicionalmente se estimaron los índices de ajuste incrementales para el CFI y el RMSEA.

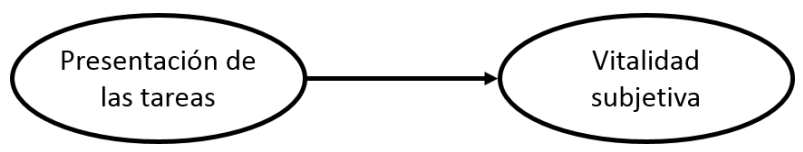

Figura 3. Modelo alternativo de mediación parcial.

Los índices de ajuste del modelo de mediación parcial (M2) fueron adecuados $\left(\chi^{2}{ }_{(474)}=906.635, \chi^{2} / g l=\right.$ 1.913, CFI $=.937$, RMSEA $=.042)$. La diferencia entre el valor de ji-cuadro del modelo hipotetizado de mediación total (M1) y el modelo de mediación parcial (M2) no resultaron estadísticamente significativas $\left(\Delta \chi^{2}=9.152 ; g l=1, p<.05\right)$. Con base a estos resultados el modelo más parsimonioso, es el de mediación parcial (M1). Los índices de ajuste incrementales resultantes de la comparación de los dos modelos $(\triangle \mathrm{CFI}=.001 ; \Delta \mathrm{RMSEA}=.001)$, indicaron diferencias de ajuste irrelevantes, siendo el modelo de mediación parcial el más parsimonioso. Finalmente, el path que representaba la relación directa entre la presentación de las tareas por parte del profesor y la vitalidad subjetiva resultó estadísticamente significativo $(\beta=.20 p<.002)$. Todo lo anterior, permite concluir que la relación entre la presentación de las tareas por parte del profesor y la vitalidad subjetiva estaba mediada por la satisfacción de la $\mathrm{NPB}$, tratándose de una mediación parcial.

\section{DISCUSIÓN}

Tomando como base los postulados de la teoría de las necesidades psicológicas básicas (Deci y Ryan, 2000), en el presente estudio se puso a prueba un modelo en que se examinaron las interrelaciones entre la presentación de las tareas realizada por el profesor, la satisfacción de las necesidades psicológicas básicas y la vitalidad subjetiva percibida por sus estudiantes en el ámbito de la educación física.

Se formularon tres hipótesis para probar el modelo propuesto, encontrando en todas ellas apoyo empírico, $\mathrm{y}$ por lo tanto, confirmando el modelo teórico hipotetizado (ver figura 1). En primer lugar, los resultados apoyaron empíricamente la asociación positiva esperada entre la percepción de los alumnos de la presentación de las tareas realizada por el profesor y la satisfacción de las NPB, lo que va acorde con investigaciones previas con atletas en el contexto deportivo (Garza-Adame et al., 2017; Tristán et al., 2016) o relacionadas con la estructura (Curran et al., 2013; Smith et al., 2015) en el deporte. Asimismo, los resultados obtenidos señalan que la forma en la que los profesores presentaron las tareas influyó sobre la satisfacción de las NPB de los alumnos. Concretamente, la satisfacción de las NPB de los alumnos mejoró cuando la presentación de las tareas presentó las siguientes características: los profesores presentaban las tareas haciendo énfasis en los elementos cualitativos críticos del movimiento deseado; comunicaban las tareas a través de claves de aprendizaje cualitativas precisas, en forma concisa, y con un número apropiado para el alumno (Landin, 1994; Masser, 1993; Rink, 2019); cuando ofrecían instrucciones verbales claras, demostraban las actividades, proporcionaban una justificación para sus solicitudes, ofrecían recomendaciones, y consideraban las perspectivas de los alumnos (Chen et al., 2012; Garza-Adame et al., 2017; Tristán et al., 2016). 


\section{Presentación de las tareas, necesidades psicológicas y vitalidad subjetiva en la educación física}



Figura 2. Solución estandarizada del modelo estructural.

De acuerdo con la segunda hipótesis de este estudio, se encontró que la percepción de satisfacción de las NPB por parte de los alumnos predijo positivamente la vitalidad subjetiva. Estos resultados concuerdan con investigaciones realizadas en el deporte (e.g., GarzaAdame et al., 2017; López-Walle et al., 2012; Tristán et al., 2016) y en el contexto de la educación física (e.g., Taylor y Lonsdale, 2010; Vlachopoulos et al., 2011). Los resultados sugieren que los alumnos se sienten vitales y llenos de energía positiva, principalmente porque sus necesidades psicológicas básicas fueron satisfechas.

De acuerdo con la tercera hipótesis del estudio, la satisfacción de las NPB medió parcialmente la relación entre la percepción de la presentación de las tareas por parte del profesor y la vitalidad subjetiva (como un indicador de bienestar). Los resultados obtenidos están en consonancia con los postulados de la SDT y los estudios realizados en el deporte (GarzaAdame et al., 2017; Tristán et al., 2016). Los resultados de este estudio aportan evidencia empírica que contribuye a confirmar la secuencia de relación entre estas variables en el contexto de la educación física, contexto en el que esta relación no había sido puesta a prueba. Los resultados obtenidos en este estudio, aportan evidencia que permite enfatizar la importancia de que los profesores de educación física presenten las tareas de forma adecuada, para así favorecer la satisfacción de las necesidades psicológicas básicas, lo que a su vez repercutirá positivamente en la percepción de vitalidad de los alumnos. Para ello, y de acuerdo a lo encontrado en la literatura, el profesor de educación física debe utilizar estrategias de presentación de las tareas que favorezcan que el alumno se sienta competente, como: realizar demostraciones completas; tener un enfoque cognitivo usando claves que sean apropiadas en número, precisas y de valor cualitativo, así como utilizar claves que sean claras para los alumnos (Garza-Adame et al., 2017; Haerens et al., 2013; Rink, 2019; Tristán et al., 2016). Para que los alumnos sientan satisfecha su necesidad de autonomía, el profesor debe buscar la participación de los alumnos en la toma de decisiones cuando sea posible, y permitir algunas opciones en términos de actividades durante la clase (Curran et al., 2013; Garza-Adame et al., 2017; Moreno-Murcia et al., 2012; Reeve y Jang, 2006; Tristán et al., 2016). Además, para que los alumnos sientan que pertenecen a la clase y que sus necesidades de relación son satisfechas, el profesor puede utilizar las siguientes estrategias en la presentación de las tareas: brindar orientación e instrucción a todos los alumnos (Haerens et al., 2013; Smith et al., 2015; Tristán et al., 2016), mencionar a los alumnos por su nombre en la explicación y demostración de las tareas, variar los participantes en los que se apoya para las demostraciones (Garza-Adame et al., 2017), e interactuar con los alumnos de una manera cálida, amistosa y afectuosa (Cox y Williams, 2008; Soenens et al., 2007).

Dentro de las limitaciones del estudio se puede señalar que no se consideró el estilo de enseñanza o modelo de instrucción empleado por el profesor. En futuras investigaciones es importante aumentar el número de 


\section{Tristán, J., Vergara-Torres, A., Vanegas-Farfano, M., Espino-Verdugo, F., Medina-Corrales, M., Tomás, I.}

alumnos e incluir alumnos de secundaria y preparatoria. También, realizar la comparación por sexo y edad de los alumnos. Asimismo, considerar aplicar un instrumento que permita determinar el estilo o modelo de instrucción utilizado por el profesor y descartar la muestra que emplee una metodología por indagación o resolución de problemas para disminuir los sesgos de los resultados. Además, considerando que no se ha estudiado la presentación de las tareas del profesor con profundidad desde la perspectiva de la SDT, sería importante continuar esta línea de investigación probando la secuencia completa de la SDT. Finalmente, se debería de probar el modelo de mediación propuesto en este estudio con una variedad de muestras compuestas por alumnos de otros países para evaluar la generalizabilidad del modelo en otros países y culturas.

\section{CONCLUSIONES}

Los resultados obtenidos permiten concluir que la presentación de las tareas por parte del profesor es una variable contextual que predice la satisfacción de las necesidades psicológicas básicas, y éstas, a su vez, predicen la vitalidad subjetiva de los alumnos de educación física participantes de este estudio. Este estudio aporta evidencia respecto a que una presentación de las tareas con calidad debe ser considerada por los profesores como un aspecto importante que impacta en la salud psicológica de los alumnos y no solamente como un aspecto pedagógico que busca el desarrollo de habilidades cognitivomotrices. Además, se obtiene evidencia empírica que apoya el papel mediador que tiene la satisfacción de las necesidades psicológicas básicas en la relación entre la presentación de las tareas y la vitalidad subjetiva. Es decir, se pone de manifiesto la importancia de que el profesor de educación física esté enfocado en satisfacer las necesidades psicológicas de los alumnos desde el proceso de planificación (tareas acordes a los intereses de los alumnos), en la clase (presentación de las tareas con calidad) y en la evaluación (participación de los alumnos en el proceso de evaluación). Este enfoque en la satisfacción de las necesidades psicológicas básicas puede repercutir positivamente en la vitalidad subjetiva de los alumnos. Es cierto que el aprendizaje y el bienestar psicológico de los alumnos no dependen únicamente de la presentación de las tareas con calidad. Sin embargo, también es cierto que, sin una adecuada habilidad para comunicar la presentación de las tareas, es muy difícil para los profesores de educación física impactar en la salud psicológica de los alumnos.

\section{APLICACIONES PRÁCTICAS}

Desde un punto de vista aplicado, los hallazgos del presente estudio subrayan la importancia que los profesores de educación física presenten las tareas de forma adecuada, y con calidad (siguiendo los consejos y estrategias presentados a lo largo del presente trabajo), siempre y cuando el modelo o metodología de enseñanza requiera una presentación de las tareas efectuada por el profesor, ya que esto puede favorecer la satisfacción de las necesidades de competencia, autonomía y relación de los alumnos, y al mismo tiempo, repercutir de forma positiva en la percepción de vitalidad de los alumnos así como, en el desarrollo de las habilidades técnicas practicadas durante las clases. Además, el conocimiento obtenido de esta investigación se puede utilizar en los programas de formación docente para contribuir en el desarrollo de habilidades de comunicación eficaces en los docentes en formación.

\section{REFERENCIAS}

1. Abad, M. T., Benito, P. J., Giménez F. J., y Robles, J. (2013). Fundamentos pedagógicos de la enseñanza comprensiva del deporte: Una revisión de la literatura. (Pedagogy of the teaching for understanding: A revision of the literature). $C C D$. Cultura_Ciencia_Deporte, 8(23), 137-146. http://dx.doi.org/10.12800/ccd.v8i23.300

2. Adie, J. W., Duda, J. L., y Ntoumanis, N. (2008). Autonomy support, basic need satisfaction and the optimal functioning of adult male and female sport participants: A test of basic needs theory. Motivation and Emotion, 32, 189-199. http://dx.doi.org/10.1007/s11031-008-9095-z

3. Bailey, R. (2006). Physical education and sport in schools: A review of benefits and outcomes. Journal of school health, 76(8), 397-401. https://doi.org/10.1111/j.17461561.2006.00132.x

4. Balaguer, I., y Castillo, I. (2007). Relationship of coach provided autonomy support to need satisfaction and well-being in young elite female tennis players. Artículo presentado en The 2007 


\section{Presentación de las tareas, necesidades psicológicas y vitalidad subjetiva en la educación física}

Annual Meeting of the International Society for Behavioral Nutrition and Physical Activity. Oslo, Norway.

5. Behzadnia, B., Adachi, P. J., Deci, E. L., y Mohammadzadeh, H. (2018). Associations between students' perceptions of physical education teachers' interpersonal styles and students' wellness, knowledge, performance, and intentions to persist at physical activity: A selfdetermination theory approach. Psychology of Sport and Exercise, 39, 10-19. https://doi.org/10.1016/j.psychsport.2018.07.003

6. Bentler, P. M. (1990). Comparative fit indexes in structural models. Psychological Bulletin, 107(2), 238. http://dx.doi.org/10.1037/0033$\underline{2909.107 .2 .238}$

7. Bentler, P. M., y Bonett, D. G. (1980). Significance tests and goodness of fit in the analysis of covariance structures. Psychological Bulletin, 88(3), 588-606.

8. Browne, M. W., y Cudeck, R. (1993). Alternative ways of assessing model fit. Sage Focus Editions, 154, 136.

9. Carmines, E., G., y McIver, J. P. (1981). Analyzing models with unobserved variables: Analysis of covariance structures. Social Measurement: Current Issues, 65-115. doi: $10.117 / 0049124183011003003$

10. Chen, F. F. (2007). Sensitivity of goodness of fit indexes to lack of measurement invariance. Structural Equation Modeling, 14, 464-504. https://doi.org/10.1080/10705510701301834.

11. Chen, W., Hendricks, K., y Archibald, K. (2011). Assessing pre-service teachers' quality teaching practices. Educational Research and Evaluation, 17(1), 13-32. https://doi.org/10.1080/13803611.2011.578008

12. Chen, W., Mason, S., Hammond-bennett, A., y Zlamout, S. (2014). Development and Validation of Assessing Quality Teaching Rubrics. Journal of Education and Training Studies, 2(3), 52-61. https://doi.org/10.11114/jets.v2i3.409

13. Chen, W., Mason, S., Staniszewski, C., Upton, A., y Valley, M. (2012). Assessing the quality of teachers' teaching practices. Educational Assessment, Evaluation and Accountability, 24(1),
25-41. https://doi.org/10.1007/s11092-011-9134-

$\underline{2}$

14. Chen, W., Zhu, W., Mason, S., HammondBennett, A., y Colombo-Dougovito, A. (2016). Effectiveness of quality physical education in improving students' manipulative skill competency. Journal of Sport and Health Science, $5(2)$, 231-238. https://doi.org/10.1016/j.jshs.2015.04.005

15. Cheung, G. W., y Rensvold, R. B. (2002). Evaluating goodness-of-fit indexes for testing measurement invariance. Structural Equation Modeling, $\quad 9(2), \quad$ 233-255. https://doi.org/10.1207/s15328007sem0902_5

16. Cox, A., y Williams, L. (2008). The Roles of Perceived Teacher Support, Motivational Climate, and Psychological Need Satisfaction in Students' Physical Education Motivation. Journal of Sport \& Exercise Psychology, 30, 222-239. doi: 10.1123/jsep.30.2.222

17. Cuevas, R., García-López, L. M., y Contreras, O. (2015). Influencia del modelo de Educación Deportiva en las necesidades psicológicas básicas. Cuadernos de Psicología del Deporte, 15(2), 155$162 . \quad$ http://dx.doi.org/10.4321/S1578$\underline{84232015000200017}$

18. Curran, T., Hill, A. P., y Niemiec, C. P. (2013). A conditional process model of children's behavioral engagement and behavioral disaffection in sport based on self-determination theory. Journal of Sport \& Exercise Psychology, 35, 30-43.

19. Deci, E. L., y Ryan, R. M. (2000). The "what" and "why" of goal pursuits: human needs and the selfdetermination of behavior. Psychological Inquiry, 11 , 227-268. http://dx.doi.org/10.1207/S15327965PLI1104_01

20. Deci, E. L., y Ryan, R. M. (2002). Handbook of Self-determination research. New York, NY: The University Rochester Press.

21. Garza-Adame, O., Tristán, J. L., Tomás, I., Hernández-Mendo, A., y López-Walle, J. M. (2017). Presentación de las tareas, satisfacción de las necesidades psicológicas básicas y bienestar en atletas universitarios. Journal of Behavior, Health \& Social Issues, 9(1), 13-20. https://doi.org/10.1016/j.jbhsi.2017.06.003 


\section{Tristán, J., Vergara-Torres, A., Vanegas-Farfano, M., Espino-Verdugo, F., Medina-Corrales, M., Tomás, I.}

22. Graham, K. C. (1988). A Qualitative Analysis of an Effective Teacher's Movement Task Presentations during a Unit of Instruction. Physical Educator, 45(4), 187-195.

23. Gusthart, J. L., y Kelly, I. W. (1993). Teachers' instructional variables in volleyball and students' improvement in motor skill. Perceptual and Motor Skills, 76, 1015-1024. doi: 10.2466/pms.1993.76.3.1015

24. Gusthart, J. L., Kelly, I. M., y Rink, J. E. (1997). The validity of the qualitative measures of teaching performance scale as a measure of teacher effectiveness. Journal of Teaching in Physical Education, 16(2), 196-210. https://doi.org/10.1123/jtpe.16.2.196

25. Haerens, L., Aelterman, N., Van den Berghe, L., De Meyer, J., Soemens, B., y Vansteenkiste, M. (2013). Observing physical education teachers' needsupportive interactions in classroom setting. Journal of Sport \& Exercise Psychology, 35, 317. https://doi.org/10.1123/jsep.35.1.3

26. Hall, T. J., Heirdon, B., y Welch, M. (2011). A Description of Preservice Teachers' task preentation skills. Physical Educator, 68(4), 188198.

27. Hu, L., y Bentler, P. M. (1995). Evaluating model fit. En R. H. Hoyle (Ed.), Structural equation modeling: Issues, concepts, and applications (pp. 76-99). Newbury Park, CA: Sage.

28. Jang, H., Reeve, J., y Deci, E. L. (2010). Engaging students in learning activities: it is not autonomy support or structure but autonomy support and structure. Journal of Educational Psychology, 102, 588-600.

http://dx.doi.org/10.1037/a0019682

29. Kelly, L. E., y Melograno, V. J. (2004). Developing the physical education curriculum: an achievement-based approach. Champaign, IL.: Human Kinetics.

30. Kwak, E. C. (2005). The Immediate Effects of Various Task Presentation Types on Middle School Students Skill Learning. International journal of applied sports sciences (IJASS), 17(1), 7-17.

31. Landin, D. (1994). The role of verbal cues in skill learning. Quest. 46 , 299-313. http://dx.doi.org/10.1080/00336297.1994.104841 $\underline{28}$

32. López-Walle, J., Balaguer, I., Castillo, I., y Tristán, J. (2012). Autonomy support basic psychological needs and well-being in Mexican athletes. The Spanish Journal of Psychology, 15(03), 1283-1292. doi: 10.5209/rev_SJOP.2012.v15.n3.39414

33. Magill, R. A. (1993). Modeling and verbal feedback influences on skill learning. International. Journal of Sport Psychology, 24(4), 358-369.

34. Masser, L. (1993). Critical Cues Help First-Grade Student's Achievement in Handstands and Forward Rolls. Journal of Teaching in Physical Education, $\quad$ 12(3), 301-312. https://doi.org/10.1123/jtpe.12.3.301

35. MacKinnon, D. P., Lockwood, C. M., y William, J. (2004). Confidence limits for the indirect effect: Distribution of the product and resampling methods. Multivariate Behavioral Research, 39, 99-128. doi:10.1207/s15327906mbr3901_4

36. McLennan, N., y Thompsom, J. (2015). Educación física de calidad (EFC): guía para los responsables politicos. Paris, Francia: UNESCO.

37. Moreno-Murcia, J. A., Cervelló, E., Montero, C., Vera J. A., y García, T. (2012). Metas sociales, necesidades psicológicas básicas y motivación intrínseca como predictores de la percepción del esfuerzo en las clases de educación física. Revista de psicología del deporte, 21(2), 0215-221.

38. Peterson, R. A. (1994). A meta-analysis of Cronbach's coefficient alpha. Journal of Consumer Research, 21(2), 381-391. https://doi.org/10.1086/209405

39. Piéron, M. (1996). Formação de professores: aquisição de técnicas de ensino e supervisã pedagógica. Facultdade de Motricidade Humana, Universidad Técnica de Lisboa, Portugal.

40. Reeve, J., y Jang, H. (2006). What teachers say and do to support students' autonomy during a learning activity. Journal of Educational Psychology, 98, 209-218. http://dx.doi.org/10.1037/0022-0663.98.1.209 


\section{Presentación de las tareas, necesidades psicológicas y vitalidad subjetiva en la educación física}

41. Reinboth, M., y Duda, J. L. (2006). Perceived motivational climate, need satisfaction and indices of well-being in team sports: A longitudinal perspective. Psychology of Sport \& Exercise, 7(3), 269-286.

https://doi.org/10.1016/j.psychsport.2005.06.002

42. Reinboth, M., Duda, J. L., y Ntoumanis, N. (2004). Dimensions of coaching behavior, need satisfaction, and the psychological and physical welfare of young athletes. Motivation and Emotion, 28(3), 297-313. doi: 10.1023/B:MOEM.0000040156.81924.b8

43. Rink, J. E. (1994). The Task Presentation in Pedagogy. Quest, 46, 270-280. https://doi.org/10.1080/00336297.1994.1048412 $\underline{6}$

44. Rink, J. E. (2019). Teaching physical education for learning. McGraw-Hill.

45. Rink, J. E. (2013). Measuring Teacher Effectiveness in Physical Education. Research Quarterly for Exercise and Sport, 84, 407-418. https://doi.org/10.1080/02701367.2014.932656

46. Rink, J. E., y Hall, T. J. (2008). Research on Effective Teaching in Elementary School Physical Education. Elementary School Journal, 108(3), 207-218. Recuperado de http://www.journals.uchicago.edu/toc/esj/current

47. Ryan, R. M., y Deci, E. L. (2000). Selfdetermination theory and the facilitation of intrinsic motivation, social development, and well-being. American Psychologist, 55, 68-78. http://dx.doi.org/10.1037/0003-066x.55.1.68

48. Ryan, R. M., y Deci, E. L. (2002). An overview of self-determination theory: An organismicdialectical perspective. En Deci, E. L. \& Ryan, R. M. (Eds.), Handbook of selfdetermination research (pp. 3-33). Rochester, NY: The University of Rochester Press.

49. Ryan, R. M., y Deci, E. L. (2017). Selfdetermination theory: Basic psychological needs in motivation, development, and wellness. New York, NY, US: Guilford Press.

50. Ryan, R. M., y Frederick, C. (1997). On energy, personality, and health: Subjective vitality as a dynamic reflection of well-being. Journal of personality,

$65(3)$,

529-565. https://doi.org/10.1111/j.14676494.1997.tb00326.x

51. Sau-Ching, H. (2001). Relationship of teacher task presentation and student learning performance in basketball. Hong Kong Journal of Sports Medicine and Sports Science, 12, 52-63.

52. Skinner, E. A., y Belmont, M. J. (1993). Motivation in the classroom: Reciprocal effects of teacher behavior and student engagement across the school year. Journal of educational psychology, 85(4), 571. doi: 10.1037/00220663.85.4.571

53. Smith, N., Tessier, D., Tzioumakis, Y., Quested, E., Appleton, P., Sarrazin, P., y Duda, J. L. (2015). Development and validation of the multidimensional motivational climate observation system. Journal of Sport \& Exercise Psychology, 37(1), 4-22. https://doi.org/10.1123/jsep.2014-0059

54. Soenens, B., Duriez, B., Vansteenkiste, M., y Goossens, L. (2007). The intergenerational transmission of empathy-related responding in adolescence: the role of maternal support. Personality and Social Psychology Bulletin, 33(3), 299-311. doi: 10.1177/0146167206296300

55. Steiger, J. H., y Lind, J. C. (1980). Statistically based tests for the number of common factors. Annual Meeting of the Psychometric Society. Iowa City, IA.

56. Taylor, I. M., y Lonsdale, C. (2010). Cultural differences in the relationships among autonomy support, psychological need satisfaction, subjective vitality, and effort in British and Chinese physical education. Journal of Sport \& Exercise Psychology, 32(5), 655-73. doi: 10.1123/jsep.32.5.655

57. Trigueros-Ramos, R., Navarro-Gómez, N., Aguilar-Parra, J. M., y León-Estrada, I. (2019). Influencia del docente de Educación Física sobre la confianza, diversión, la motivación y la intención de ser físicamente activo en la adolescencia. Cuadernos de Psicología del Deporte, 19(1), 222-232

58. Tristán, J., López-Walle, J., Tomás, I., PérezGarcía, J. A., Cantú-Berrueto, A., y Castillo, I. (2016). Development and validation of the Coach's Task Presentation Scale: A self-report 
Tristán, J., Vergara-Torres, A., Vanegas-Farfano, M., Espino-Verdugo, F., Medina-Corrales, M., Tomás, I.

instrument. Psychology of Sport \& Exercise, 25, 68-77.

http://dx.doi.org/10.1016/j.psychsport.2016.04.00 $\underline{2}$

59. Tucker, C. M., Zayco, R. A., Herman, K. C., Reinke, W. M., Trujillo, M., Carraway, K., . . . Ivery, P. D. (2002). Teacher and child variables as predictors of academic engagement among lowincome African American children. Psychology in the Schools, 39(4), 477- 488. doi: $10.1002 /$ pits. 10038

60. Vlachopoulos, S. P., Katartzi, E. S., y Kontou, M. G. (2011). The Basic Psychological Needs in Physical Education Scale. Journal of Teaching in Physical Education, 30(3), 263-280. http://dx.doi.org/10.1123/jtpe.30.3.263

61. Wheaton, D. E., Muthén, B., Alwin, D. F., y Summers, G. F. (1977). Assessing reliability and stability in panel models. Sociological Methodology, 8, 84-136. doi: 10.2307/270754

62. William, J., y MacKinnon, D. P. (2008). Resampling and distribution of the product methods for testing indirect effects in complex models. Structural Equation Modeling, 15, 23-52. doi:10.1080/10705510701758166

63. Zamarripa, J., Castillo, I., Tomás, I., Delgado, M., y Álvarez, O. (2017). Escala Mexicana de Satisfacción de las Necesidades Psicológicas Básicas en Educación Física. Revista de Psicología del Deporte, 26, 79-84. Recuperado de http://www.redalyc.org/articulo.oa?id=23515204 $\underline{5011}$ 